\title{
Biotech marks time in Q3
}

Stacy Lawrence

In a risk-averse market, biotech stock market indices showed sluggish growth, being outperformed by broader market indicators. Venture capital funding remained relatively stable but public biotechs were able to

take advantage of debt, private investment in public equitities (PIPEs)

\section{Biotechnology stock market performance}

US biotech indices performed better at the end of 3Q05; the Swiss Exchange continued its positive growth

$\square$ Biocentury Biotech $100 \square$ Dow Jones $\square$ Nasdaq $\square$ Nasdaq Biotech $\square$ Swiss Exchange

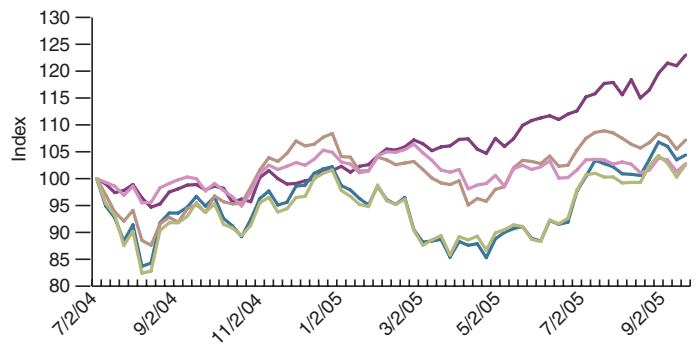

Source: Multex, BioCentury

\section{Global biotech industry financing}

Compared with the past 18 months, funding overall, but particularly debt funding, PIPEs and follow-on financings, is up.

$\square$ IPOs $\square$ PIPES $\square$ Venture capital $\square$ Follow-on financing $\square$ Debt and other financing $\square$ Partnering

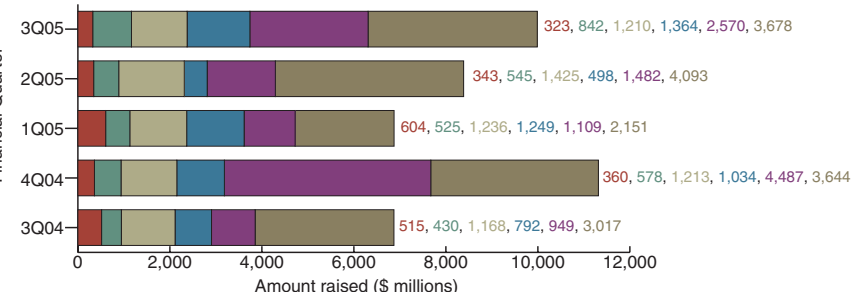

Source: Biocentury, Burrill \& Company and follow-on financings to boost available cash. Funds raised through debt increased by three-quarters over the total in Q2; the amount raised through PIPEs increased by half the prior quarter's total; follow-on funding almost tripled.

\section{Global biotech initial public offerings}

The IPO market in the US rebounded somewhat; Europe's recent IPO honeymoon came to an end. Total amount raised was the smallest since 3Q03.

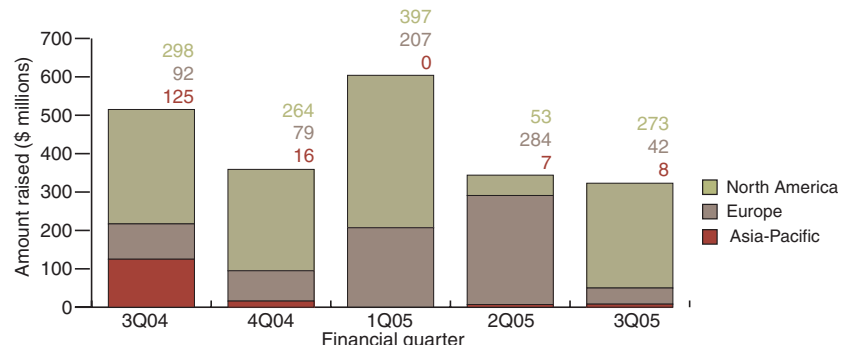

Number of IPOs

North Americ

Europe

Asia-Pacific

Source: BioCentury

\section{Global biotech venture capital investment}

Biotech VC funding remains relatively stable; activity in Asia is increasing slightly.

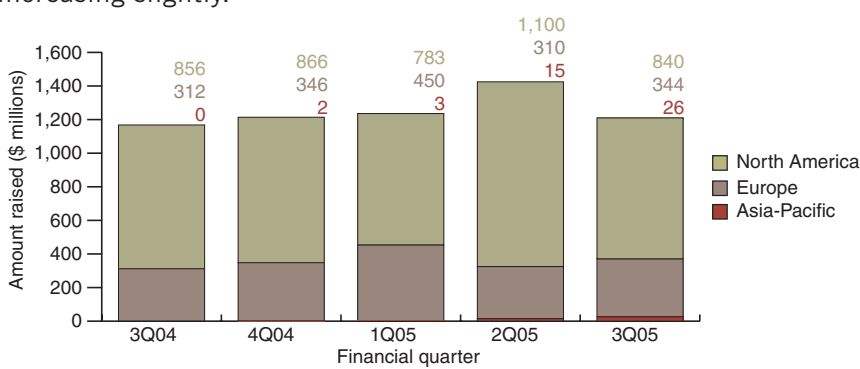

Source: BioCentury

\begin{tabular}{|c|c|c|c|c|}
\hline 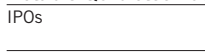 & Company (lead underwriter) & Amount raised(\$ millions) & $\begin{array}{l}\text { Percent change in stock price } \\
\text { since offer }{ }^{1}\end{array}$ & Date launched \\
\hline & Coley Pharmaceutical (Merrill Lynch, JP Morgan) & 110.40 & $14 \%$ & 10-Aug \\
\hline & Genomic Health (JP Morgan, Lehman Brothers) & 60.20 & $-4 \%$ & 28-Sep \\
\hline & Sunesis Pharmaceuticals (Lehman Brothers, SG Cowen) & 42.00 & $-4 \%$ & 27-Sep \\
\hline & Advanced Life Sciences (C.E. Unterberg Towbin) & 32.00 & $-1 \%$ & 5-Aug \\
\hline & Avalon Pharmaceuticals (W.R. Hambrecht) & 28.88 & $-12 \%$ & 29-Sep \\
\hline \multirow[t]{5}{*}{ Venture Capital } & Company (Lead investors) & Amount invested (\$ millions) & Round number & Date closed \\
\hline & Replidyne (Duquesne Capital, Healthcare Investment Partners, MDS Capital) & 62.5 & 4 & 8-Sep \\
\hline & Affymax (JAFCO, Bear Stearns) & 60 & 4 & 18-Jul \\
\hline & Amicus Therapeutics (Quaker BioVentures) & 55 & 3 & 8-Sep \\
\hline & Cerexa (Frazier Healthcare Ventures, New Leaf Venture Partners) & 50 & 2 & 23-Aug \\
\hline \multirow[t]{6}{*}{ Mergers and acquisitions } & Target & Acquirer & Value ( $\$$ millions) & Date announced \\
\hline & IVAX & Teva Pharmaceutical & 7,400 & 25-Jul \\
\hline & Chiron & Novartis $^{*}$ & 4,500 & 5-Sep \\
\hline & ID Biomedical & GlaxoSmithKline & 1,400 & 7-Sep \\
\hline & Eyetech Pharmaceuticals & OSI Pharmaceuticals & 935 & 21-Aug \\
\hline & LabOne & Quest Diagnostics & 934 & 8-Aug \\
\hline \multirow[t]{8}{*}{ Licensing/collaboration } & Researcher & Investor & Value ( $\$$ millions) & Deal type \\
\hline & Protein Design Labs & Biogen IDEC & 800 & Collaboration, development \\
\hline & Alnylam Pharmaceuticals & Novartis & 700 & Collaboration, discovery and development \\
\hline & Emory University & $\begin{array}{l}\text { Gilead Sciences, Royalty } \\
\text { Pharma }\end{array}$ & 525 & Royalty interest \\
\hline & Avanir Pharmaceuticals & AstraZeneca & 340 & Collaboration, discovery and development \\
\hline & Astex Therapeutics & AstraZeneca & 275 & Collaboration, discovery and development \\
\hline & Sirna Therapeutics & Allergan & 250 & Collaboration, development \\
\hline & Adherex Technologies & GlaxoSmithKline & 223 & License \\
\hline
\end{tabular}

